\title{
INTERVAL OSCILLATION CRITERIA FOR HIGHER ORDER NEUTRAL NONLINEAR DIFFERENTIAL EQUATIONS WITH DEVIATING ARGUMENTS
}

\author{
Run Xu, Fanwei Meng
}

\begin{abstract}
Some oscillation criteria for $n$th order neutral differential equations with deviating arguments of the form

$\left[r(t)\left|(y(t)+p(t) y(\tau(t)))^{(n-1)}\right|^{\alpha-1}(y(t)+p(t) y(\tau(t)))^{(n-1)}\right]^{\prime}+\sum_{i=1}^{m} q_{i}(t) f_{i}\left(y\left(\sigma_{i}(t)\right)\right)=0$

$n$ even are established. New oscillation criteria are different from most known ones in the sense that they based on a class of new function $H(t, s)$ defined in the sequel. The results are sharper than some previous results which can be seen by the examples at the end of this paper.
\end{abstract}

\section{INTRODUCTION}

In this paper we consider the oscillation behavior of solutions of the $n$-th order neutral differential equations of the form

$$
\begin{aligned}
{\left[r(t)\left|(y(t)+p(t) y(\tau(t)))^{(n-1)}\right|^{\alpha-1}(y(t)+p(t) y(\tau(t)))^{(n-1)}\right]^{\prime} } & \\
& +\sum_{i=1}^{m} q_{i}(t) f_{i}\left(y\left(\sigma_{i}(t)\right)\right)=0,
\end{aligned}
$$

Received 07-10-2008, Accepted 27-12-2009.

2000 Mathematics Subject Classification: 34C10.

Key words and Phrases: Oscillation; Even Order; Nonlinear Differential Equations; Neutral type.

This research was partial supported by the NNSF of China(10771118) and EDF of shandong Province, China(J07yh05) 
where $t \geq t_{0}, n \geq 2$ is even integer, $\alpha>0$ are constant. In this paper, we assume that

$\left(I_{1}\right) p(t) \in C\left(\left[t_{0}, \infty\right) ;[0,1)\right), q_{i}(t) \in C\left(\left[t_{0}, \infty\right) ;[0, \infty)\right), f_{i} \in C(R ; R), i=1,2, \cdots, m$, $t \in\left[t_{0}, \infty\right)$.

$\left(I_{2}\right) r(t) \in C^{1}\left(\left[t_{0}, \infty\right) ;(0, \infty)\right), r^{\prime}(t) \geq 0, R_{1}(t):=\int_{t_{0}}^{t} \frac{d s}{r^{\frac{1}{\alpha}}(s)} \rightarrow \infty(t \rightarrow \infty)$.

$\left(I_{3}\right) \frac{f_{i}(x)}{|x|^{\alpha-1} x} \geq \beta_{i}>0$ for $x \neq 0, \beta_{i}$ are constants, $i=1,2, \cdots, m$.

$\left(I_{4}\right) \tau(t), \sigma_{i}(t) \in C^{1}\left(\left[t_{0}, \infty\right) ;[0, \infty)\right), \tau(t) \leq t, \sigma_{i}(t) \leq t, \sigma^{\prime}(t)>0$ for $t \geq t_{0}$ and $\lim _{t \rightarrow \infty} \sigma_{i}(t)=\lim _{t \rightarrow \infty} \sigma(t)=\lim _{t \rightarrow \infty} \tau(t)=\infty, i=1,2, \cdots, m$, where $\sigma(t) \leq$ $\min \left\{\sigma_{1}(t), \sigma_{2}(t), \cdots, \sigma_{m}(t), \frac{t}{2}\right\}$.

By a solution of Eq. (1), we mean a function $y(t) \in C^{n-1}\left(\left[T_{x}, \infty\right) ; R\right)$ for some $T_{x} \geq t_{0}$ which has the property that

$$
r(t)\left|(y(t)+p(t) y(\tau(t)))^{(n-1)}\right|^{\alpha-1}(y(t)+p(t) y(\tau(t)))^{(n-1)} \in C^{1}\left(\left[T_{x}, \infty\right) ; R\right)
$$

and satisfies Eq. (1) on $\left[T_{x}, \infty\right)$.

A nontrivial solution of Eq. (1) is called oscillatory if it has arbitrary large zero. Otherwise, it is called nonoscillatory. Eq. (1) is called oscillatory if all of its solutions are oscillatory.

If $p(t)=0, r(t)=1, m=1$, then Eq. (1) becomes

$$
\left(\left|x^{(n-1)}(t)\right|^{\alpha-1} x^{(n-1)}(t)\right)^{\prime}+q(t) f(x(\sigma(t)))=0
$$

and the related equations have been studied by Agarwal et. al. [2], Xu et. al. [15].

Eq. (1) with $n=2, p(t)=0, m=1$, namely, the equation

$$
\left[r(t)\left|x^{\prime}(t)\right|^{\alpha-1} x^{\prime}(t)\right]^{\prime}+q(t) f(x(\sigma(t)))=0
$$

and related equations have been investigated by Dzurina and Stavroulakis [4], Sun and Meng [14], Mirzov [10-12], Elbert [5,6] Agarwal et. al. [1], Chern et. al. [3], Li [7], Zhuang and Li [19].

Recently, Xu and Meng [16-18] have studied the oscillation properties of Eq. (1) for $n=2$. Very recently Meng and $\mathrm{Xu}[8,9]$ have investigated the oscillation properties for higher order neutral differential equations.

Motivated by the idea of $\mathrm{Li}$ [7], by using averaging functions and inequality, in this paper we obtain several new interval criteria for oscillation, that is, criteria are given by the behavior of Eq. (1) (or of $r, p$ and $q_{i}$ ) only on a sequence of subintervals of $[t, \infty)$. Our results improve and extend the results of $\mathrm{Li}[7]$ and Zhuang and $\mathrm{Li}$ [19]. In order to prove our Theorems, we use the function class $X$ to study the oscillatory of Eq. (1). We say that a function $H=H(t, s)$ belongs to the function class $X$, if $H \in C\left(D ; R_{+}\right)$, where $D=\left\{(t, s): t_{0} \leq s \leq t<\infty\right\}$, which 
satisfies $H(t, t)=0, H(t, s)>0$ for $t>s$, and has partial derivative $\frac{\partial H}{\partial s}$ and $\frac{\partial H}{\partial t}$ on $\mathrm{D}$ such that

$$
\frac{\partial H}{\partial t}=h_{1}(t, s) \sqrt{H(t, s)}, \frac{\partial H}{\partial s}=-h_{2}(t, s) \sqrt{H(t, s)},
$$

where $h_{1}(t, s), h_{2}(t, s)$ are locally nonnegative continuous functions on $D$.

\section{MAIN RESULTS}

First, we give the following lemmas for our results.

Lemma 2.1. [13] Let $u(t) \in C^{n}\left(\left[t_{0}, \infty\right) ; R^{+}\right)$. If $u^{(n)}(t)$ is eventually of one sign for all large $t$, say $t_{1}>t_{0}$, then there exist $a t_{x}>t_{0}$ and an integer $l, 0 \leq l \leq n$, with $n+l$ even for $u^{(n)}(t) \geq 0$ or $n+l$ odd for $u^{(n)}(t) \leq 0$ such that $l>0$ implies that $u^{(k)}(t)>0$ for $t>t_{x}, k=0,1,2, \cdots, l-1$, and $l \leq n-1$ implies that $(-1)^{l+k} u^{(k)}(t)>0$ for $t>t_{x}, k=l, l+1, \cdots, n-1$.

Lemma 2.2. [13] If the function $u(t)$ is as in Lemma 2.1 and $u^{(n-1)}(t) u^{(n)}(t) \leq 0$ for $t>t_{x}$, then there exists a constant $\theta, 0<\theta<1$, such that

$$
u(t) \geq \frac{\theta}{(n-1) !} t^{n-1} u^{(n-1)}(t) \text { for all large } t .
$$

and

$$
u^{\prime}\left(\frac{t}{2}\right) \geq \frac{\theta}{(n-2) !} t^{n-2} u^{(n-1)}(t) \text { for all large } t
$$

Lemma 2.3. Suppose that $y(t)$ is an eventually positive solution of Eq. (1), let

$$
z(t)=y(t)+p(t) y(\tau(t))
$$

then there exists a number $t_{1} \geq t_{0}$ such that

$$
z(t)>0, z^{\prime}(t)>0, z^{(n-1)}(t)>0 \quad \text { and } \quad z^{(n)}(t) \leq 0, t \geq t_{1} .
$$

Proof. Since $y(t)$ is an eventually positive solution of $(1)$, from $\left(I_{4}\right)$, there exists a number $t_{1} \geq t_{0}$ such that

$$
y(t)>0, y(\tau(t))>0, y\left(\sigma_{i}(t)\right)>0, t \geq t_{1} .
$$


Noting that $p(t) \geq 0$, we have $z(t)>0, t \geq t_{1}$ and from $\left(I_{1}\right),\left(I_{3}\right)$ we have

$$
\left(r(t)\left|z^{(n-1)}(t)\right|^{\alpha-1} z^{(n-1)}(t)\right)^{\prime}=-\sum_{i=1}^{m} q_{i}(t) f_{i}\left(y\left(\sigma_{i}(t)\right)\right) \leq 0, t \geq t_{1} .
$$

So $r(t)\left|z^{(n-1)}(t)\right|^{\alpha-1} z^{(n-1)}(t)$ is decreasing and $z^{(n-1)}(t)$ is eventually of one sign. we claim that

$$
z^{(n-1)}(t) \geq 0 \text { for } t \geq t_{1}
$$

Otherwise, if there exist a $\widetilde{t_{1}} \geq t_{1}$ such that $z^{(n-1)}\left(\widetilde{t_{1}}\right)<0$, then for all $t \geq \widetilde{t_{1}}$,

$$
r(t)\left|z^{(n-1)}(t)\right|^{\alpha-1} z^{(n-1)}(t) \leq r\left(\widetilde{t_{1}}\right)\left|z^{(n-1)}\left(\widetilde{t}_{1}\right)\right|^{\alpha-1} z^{(n-1)}\left(\widetilde{t}_{1}\right)=-C(C>0),
$$

then we have $-z^{(n-1)}(t) \geq\left(\frac{C}{r(t)}\right)^{\frac{1}{\alpha}}, t \geq \tilde{t_{1}}$, integrating the above inequality from $\widetilde{t_{1}}$ to $t$, we have

$$
z^{(n-2)}(t) \leq z^{(n-2)}\left(\widetilde{t_{1}}\right)-C^{\frac{1}{\alpha}}\left(R(t)-R\left(\widetilde{t_{1}}\right)\right) .
$$

Letting $t \rightarrow \infty$, from $\left(I_{2}\right)$, we get $\lim _{t \rightarrow \infty} z^{(n-2)}(t)=-\infty$, which implies $z^{(n-1)}(t)$ and $z^{(n-2)}(t)$ are negative for all large $t$, from Lemma 2.1, no two consecutive derivatives can be eventually negative, for this would imply that $\lim _{t \rightarrow \infty} z(t)=-\infty$, a contradiction. Hence $z^{(n-1)}(t) \geq 0$ for $t \geq t_{1}$. from Eq. (1) and $\left(I_{1}\right),\left(I_{2}\right)$ we have

$$
\begin{aligned}
& \alpha r(t)\left(z^{(n-1)}(t)\right)^{\alpha-1} z^{(n)}(t)=\left[r(t)\left(z^{(n-1)}(t)\right)^{\alpha}\right]^{\prime}-r^{\prime}(t)\left(z^{(n-1)}(t)\right)^{\alpha} \\
= & -\sum_{i=1}^{m} q_{i}(t) f_{i}\left(y\left(\sigma_{i}(t)\right)\right)-r^{\prime}(t)\left(z^{(n-1)}(t)\right)^{\alpha} \leq 0, t \geq t_{1},
\end{aligned}
$$

this implies that $z^{(n)}(t) \leq 0, t \geq t_{1}$. From Lemma 2.1 again (note $n$ is even), we have $z^{\prime}(t)>0, t \geq t_{1}$. This completes the proof.

Theorem 2.1. Assume that there exist a positive, nondecreasing function $\rho(t) \in$ $C^{1}\left(\left[t_{0}, \infty\right)\right)$ such that for any constant $M>0$, some $H \in X$ and for each sufficient large $T_{0} \geq t_{0}$, there exist increasing divergent sequences of positive numbers $\left\{a_{n}\right\},\left\{b_{n}\right\},\left\{c_{n}\right\}$ with $T_{0} \leq a_{n}<c_{n}<b_{n}$ such that

$$
\begin{aligned}
& \frac{1}{H\left(c_{n}, a_{n}\right)} \int_{a_{n}}^{c_{n}} H\left(s, a_{n}\right) \rho(s) C_{1}(s) d s+\frac{1}{H\left(b_{n}, c_{n}\right)} \int_{c_{n}}^{b_{n}} H\left(b_{n}, s\right) \rho(s) C_{1}(s) d s \\
> & \frac{1}{H\left(c_{n}, a_{n}\right)} \int_{a_{n}}^{c_{n}}\left[h_{1}\left(s, a_{n}\right)+\sqrt{H\left(s, a_{n}\right)} \frac{\rho^{\prime}(s)}{\rho(s)}\right]^{\alpha+1} \frac{C_{2}(s)}{H^{\frac{\alpha-1}{2}}\left(s, a_{n}\right)} d s \\
+ & \frac{1}{H\left(b_{n}, c_{n}\right)} \int_{c_{n}}^{b_{n}}\left[h_{2}\left(b_{n}, s\right)+\sqrt{H\left(b_{n}, s\right)} \frac{\rho^{\prime}(s)}{\rho(s)}\right]^{\alpha+1} \frac{C_{2}(s)}{H^{\frac{\alpha-1}{2}}\left(b_{n}, s\right)} d s,
\end{aligned}
$$


where

$$
C_{1}(t)=\sum_{i=1}^{m} \beta_{i} q_{i}(t)\left(1-p\left(\sigma_{i}(t)\right)\right)^{\alpha}, C_{2}(t)=\frac{r(t) \rho(t)}{M^{\alpha}(\alpha+1)^{\alpha+1}\left(\sigma^{\prime}(t) \sigma^{n-2}(t)\right)^{\alpha}},
$$

then every solution of Eq. (1) is oscillatory.

Proof. Suppose the contrary, let $y(t)$ is a nonoscillatory solution of Eq. (1), without loss of generality we assume

$$
y(t)>0, y(\tau(t))>0 \quad \text { for } \quad t \geq t_{1} \geq t_{0} .
$$

Then

$$
z(t)=y(t)+p(t) y(\tau(t))>0 \quad \text { for } \quad t \geq t_{1} \geq t_{0} .
$$

From Lemma 2.3, there exists $t_{2} \geq t_{1}$ such that

$$
z(t)>0, z^{\prime}(t)>0, z^{(n-1)}(t)>0 \text { and } z^{(n)}(t) \leq 0, t \geq t_{2}
$$

It is easy to check that we can apply Lemma 2.2 and conclude that there exist $0<\theta<1$ and $t_{3}>t_{2}$ such that

$$
\begin{aligned}
z^{\prime}(\sigma(t)) & \geq \frac{\theta}{(n-2) !}(2 \sigma(t))^{n-2} z^{(n-1)}(2 \sigma(t)) \\
& \geq \frac{\theta}{(n-2) !} 2^{n-2} \sigma^{n-2}(t) z^{(n-1)}(t)=M \sigma^{n-2}(t) z^{(n-1)}(t), t \geq t_{3},
\end{aligned}
$$

where $M=\frac{\theta}{(n-2) !} 2^{n-2}$.

From (5), we have

$$
y(t)=z(t)-p(t) y(\tau(t)) \geq z(t)-p(t) z(\tau(t)) \geq z(t)(1-p(t)), t \geq t_{3} .
$$

Since $\lim _{t \rightarrow \infty} \sigma(t)=\infty$, there exists $t_{4} \geq t_{3}$ such that $\sigma(t) \geq t_{3}, t \geq t_{4}$, so

$$
y(\sigma(t)) \geq z(\sigma(t))(1-p(\sigma(t))), t \geq t_{4}
$$

By $\left(I_{3}\right)$ and $(15)$ we get

$$
f_{i}\left(y\left(\sigma_{i}(t)\right)\right) \geq \beta_{i} y^{\alpha}\left(\sigma_{i}(t)\right) \geq \beta_{i} z^{\alpha}\left(\sigma_{i}(t)\right)\left(1-p\left(\sigma_{i}(t)\right)\right)^{\alpha}, t \geq t_{4} .
$$

From (1), (16), we get 


$$
\begin{aligned}
0 & =\left[r(t)\left(z^{(n-1)}(t)\right)^{\alpha}\right]^{\prime}+\sum_{i=1}^{m} \beta_{i} q_{i}(t) f_{i}\left(y\left(\sigma_{i}(t)\right)\right) \\
& \geq\left[r(t)\left(z^{(n-1)}(t)\right)^{\alpha}\right]^{\prime}+\sum_{i=1}^{m} \beta_{i} q_{i}(t) z^{\alpha}\left(\sigma_{i}(t)\right)\left(1-p\left(\sigma_{i}(t)\right)\right)^{\alpha} \\
& \geq\left[r(t)\left(z^{(n-1)}(t)\right)^{\alpha}\right]^{\prime}+\sum_{i=1}^{m} \beta_{i} q_{i}(t) z^{\alpha}(\sigma(t))\left(1-p\left(\sigma_{i}(t)\right)\right)^{\alpha}, t \geq t_{4} .
\end{aligned}
$$

Let

$$
w(t)=\rho(t) \frac{r(t)\left(z^{(n-1)}(t)\right)^{\alpha}}{z^{\alpha}(\sigma(t))}, t \geq t_{4},
$$

clearly, $w(t)>0$, from (13), (17) and (18) we get

$$
\begin{aligned}
w^{\prime}(t)= & \frac{\rho^{\prime}(t)}{\rho(t)} w(t)+\rho(t) \frac{\left[r(t)\left(z^{(n-1)}(t)\right)^{\alpha}\right]^{\prime}}{z^{\alpha}(\sigma(t))} \\
& -\rho(t) \frac{r(t)\left(z^{(n-1)}(t)\right)^{\alpha} \alpha z^{\alpha-1}(\sigma(t)) z^{\prime}(\sigma(t)) \sigma^{\prime}(t)}{z^{2 \alpha}(\sigma(t))} \\
\leq & \frac{\rho^{\prime}(t)}{\rho(t)} w(t)-\rho(t) \sum_{i=1}^{m} \beta_{i} q_{i}(t)\left(1-p\left(\sigma_{i}(t)\right)\right)^{\alpha} \\
& -\alpha \sigma^{\prime}(t) \rho(t) \frac{r(t)\left(z^{(n-1)}(t)\right)^{\alpha} z^{\prime}(\sigma(t))}{z^{\alpha+1}(\sigma(t))}, \\
\leq & \frac{\rho^{\prime}(t)}{\rho(t)} w(t)-\rho(t) \sum_{i=1}^{m} \beta_{i} q_{i}(t)\left(1-p\left(\sigma_{i}(t)\right)\right)^{\alpha} \\
& -\alpha M \sigma^{\prime}(t) \sigma^{n-2}(t) \frac{w^{\frac{\alpha+1}{\alpha}}(t)}{(r(t) \rho(t))^{\frac{1}{\alpha}}} \\
\leq & \frac{\rho^{\prime}(t)}{\rho(t)} w(t)-\rho(t) C_{1}(t)-\alpha M \sigma^{\prime}(t) \sigma^{n-2}(t) \frac{w^{\frac{\alpha+1}{\alpha}}(t)}{(r(t) \rho(t))^{\frac{1}{\alpha}}} .
\end{aligned}
$$

Then from above inequality we have

$$
\rho(t) C_{1}(t) \leq-w^{\prime}(t)+\frac{\rho^{\prime}(t)}{\rho(t)} w(t)-\alpha M \sigma^{\prime}(t) \sigma^{n-2}(t) \frac{w^{\frac{\alpha+1}{\alpha}}(t)}{(r(t) \rho(t))^{\frac{1}{\alpha}}} .
$$

Multiplying (19) by $H(s, t)$, integrating it with respect $s$ from $t$ to $c_{n}$ and 
using (4) we get that

$$
\begin{aligned}
\int_{t}^{c_{n}} H(s, t) \rho(s) C_{1}(s) d s \leq & -\int_{t}^{c_{n}} w^{\prime}(s) H(s, t) d s+\int_{t}^{c_{n}} H(s, t) \frac{\rho^{\prime}(s)}{\rho(s)} w(s) d s \\
& -\alpha M \int_{t}^{c_{n}} H(s, t) \sigma^{\prime}(s) \sigma^{n-2}(s) \frac{w^{\frac{\alpha+1}{\alpha}}(s)}{(r(s) \rho(s))^{\frac{1}{\alpha}}} d s \\
= & -H\left(c_{n}, t\right) w\left(c_{n}\right)+\int_{t}^{c_{n}} w(s) h_{1}(s, t) \sqrt{H(s, t)} d s \\
& +\int_{t}^{c_{n}} H(s, t) \frac{\rho^{\prime}(s)}{\rho(s)} w(s) d s \\
& -\alpha M \int_{t}^{c_{n}} H(s, t) \sigma^{\prime}(s) \sigma^{n-2}(s) \frac{w^{\frac{\alpha+1}{\alpha}}(s)}{(r(s) \rho(s))^{\frac{1}{\alpha}}} d s \\
= & -H\left(c_{n}, t\right) w\left(c_{n}\right) \\
& +\int_{t}^{c_{n}}\left[h_{1}(s, t) \sqrt{H(s, t)}+H(s, t) \frac{\rho^{\prime}(s)}{\rho(s)}\right] w(s) d s \\
& -\alpha M \int_{t}^{c_{n}} H(s, t) \sigma^{\prime}(s) \sigma^{n-2}(s) \frac{w^{\frac{\alpha+1}{\alpha}}(s)}{(r(s) \rho(s))^{\frac{1}{\alpha}}} d s
\end{aligned}
$$

Set

$$
F(w)=\left[h_{1} \sqrt{H}+H \frac{\rho^{\prime}}{\rho}\right] w-\alpha M H \sigma^{\prime} \sigma^{n-2} \frac{w^{\frac{\alpha+1}{\alpha}}}{(r \rho)^{\frac{1}{\alpha}}},
$$

by simple calculate, we can get that when

$$
w=\frac{\left(h_{1} \sqrt{H}+H \frac{\rho^{\prime}}{\rho}\right)^{\alpha} r \rho}{\left[M(\alpha+1) H \sigma^{\prime} \sigma^{n-2}\right]^{\alpha}},
$$

$F(w)$ has the maximum value

$$
\frac{\left(h_{1} \sqrt{H}+H \frac{\rho^{\prime}}{\rho}\right)^{\alpha+1} r \rho}{\left[M(\alpha+1) H \sigma^{\prime} \sigma^{n-2}\right]^{\alpha}(\alpha+1)},
$$

that is

$$
F(w) \leq F_{\max }(w)=\left(h_{1}+\sqrt{H} \frac{\rho^{\prime}}{\rho}\right)^{\alpha+1} H^{\frac{1-\alpha}{2}} C_{2}(s),
$$

from above inequality, we get

$$
\begin{aligned}
& \int_{t}^{c_{n}} H(s, t) \rho(s) C_{1}(s) d s \leq-H\left(c_{n}, t\right) w\left(c_{n}\right) \\
+ & \int_{t}^{c_{n}}\left(h_{1}(s, t)+\sqrt{H(s, t)} \frac{\rho^{\prime}(s)}{\rho(s)}\right)^{\alpha+1} H^{\frac{1-\alpha}{2}}(s, t) C_{2}(s) d s .
\end{aligned}
$$


Letting $t \rightarrow a_{n}^{+}$in the above, we obtain

$$
\begin{aligned}
& \frac{1}{H\left(c_{n}, a_{n}\right)} \int_{a_{n}}^{c_{n}} H\left(s, a_{n}\right) \rho(s) C_{1}(s) d s \leq-w\left(c_{n}\right) \\
& \quad+\frac{1}{H\left(c_{n}, a_{n}\right)} \int_{a_{n}}^{c_{n}}\left[h_{1}\left(s, a_{n}\right)+\sqrt{H\left(s, a_{n}\right)} \frac{\rho^{\prime}(s)}{\rho(s)}\right]^{\alpha+1} \frac{C_{2}(s)}{H^{\frac{\alpha-1}{2}}\left(s, a_{n}\right)} d s .
\end{aligned}
$$

Multiplying (19) by $H(t, s)$, integrating it with respect $s$ from $c_{n}$ to $t$, using (4) and by simple calculate we get that

$$
\begin{aligned}
\int_{c_{n}}^{t} H(t, s) \rho(s) C_{1}(s) d s \leq & -\int_{c_{n}}^{t} w^{\prime}(s) H(t, s) d s+\int_{c_{n}}^{t} H(t, s) \frac{\rho^{\prime}(s)}{\rho(s)} w(s) d s \\
& -\alpha M \int_{c_{n}}^{t} H(t, s) \sigma^{\prime}(s) \sigma^{n-2}(s) \frac{w^{\frac{\alpha+1}{\alpha}}(s)}{(r(s) \rho(s))^{\frac{1}{\alpha}}} d s \\
= & H\left(t, c_{n}\right) w\left(c_{n}\right)-\int_{c_{n}}^{t} w(s) h_{2}(t, s) \sqrt{H(t, s)} d s \\
& +\int_{c_{n}}^{t} H(t, s) \frac{\rho^{\prime}(s)}{\rho(s)} w(s) d s \\
& -\alpha M \int_{c_{n}}^{t} H(t, s) \sigma^{\prime}(s) \sigma^{n-2}(s) \frac{w^{\frac{\alpha+1}{\alpha}}(s)}{(r(s) \rho(s))^{\frac{1}{\alpha}}} d s \\
\leq & H\left(t, c_{n}\right) w\left(c_{n}\right) \\
& +\int_{c_{n}}^{t}\left[h_{2}(t, s) \sqrt{H(t, s)}+H(t, s) \frac{\rho^{\prime}(s)}{\rho(s)}\right] w(s) d s \\
& -\alpha M \int_{c_{n}}^{t} H(t, s) \sigma^{\prime}(s) \sigma^{n-2}(s) \frac{w^{\frac{\alpha+1}{\alpha}}(s)}{(r(s) \rho(s))^{\frac{1}{\alpha}}} d s \\
\leq & H\left(t, c_{n}\right) w\left(c_{n}\right) \\
& +\int_{c_{n}}^{t}\left[h_{2}(t, s)+\sqrt{H(t, s)} \frac{\rho^{\prime}(s)}{\rho(s)}\right]^{\alpha+1} H^{\frac{1-\alpha}{2}}(t, s) C_{2}(s) d s .
\end{aligned}
$$

Letting $t \rightarrow b_{n}^{+}$in the above, we obtain

$$
\begin{aligned}
& \frac{1}{H\left(b_{n}, c_{n}\right)} \int_{c_{n}}^{b_{n}} H\left(b_{n}, s\right) \rho(s) C_{1}(s) d s \leq w\left(c_{n}\right) \\
& \quad+\frac{1}{H\left(b_{n}, c_{n}\right)} \int_{c_{n}}^{b_{n}}\left[h_{2}\left(b_{n}, s\right)+\sqrt{H\left(b_{n}, s\right)} \frac{\rho^{\prime}(s)}{\rho(s)}\right]^{\alpha+1} \frac{C_{2}(s)}{H^{\frac{\alpha-1}{2}}\left(b_{n}, s\right)} d s
\end{aligned}
$$


Adding (20) and (21) we have the inequality

$$
\begin{aligned}
& \frac{1}{H\left(c_{n}, a_{n}\right)} \int_{a_{n}}^{c_{n}} H\left(s, a_{n}\right) \rho(s) C_{1}(s) d s+\frac{1}{H\left(b_{n}, c_{n}\right)} \int_{c_{n}}^{b_{n}} H\left(b_{n}, s\right) \rho(s) C_{1}(s) d s \\
\leq & \frac{1}{H\left(c_{n}, a_{n}\right)} \int_{a_{n}}^{c_{n}}\left[h_{1}\left(s, a_{n}\right)+\sqrt{H\left(s, a_{n}\right)} \frac{\rho^{\prime}(s)}{\rho(s)}\right]^{\alpha+1} \frac{C_{2}(s)}{H^{\frac{\alpha-1}{2}}\left(s, a_{n}\right)} d s \\
+ & \frac{1}{H\left(b_{n}, c_{n}\right)} \int_{c_{n}}^{b_{n}}\left[h_{2}\left(b_{n}, s\right)+\sqrt{H\left(b_{n}, s\right)} \frac{\rho^{\prime}(s)}{\rho(s)}\right]^{\alpha+1} \frac{C_{2}(s)}{H^{\frac{\alpha-1}{2}}\left(b_{n}, s\right)} d s, t \geq t_{4} .
\end{aligned}
$$

Which contradict the assumption (10). Thus, the claim holds, i.e., no nontrivial solution of Eq. (1) can be eventually positive. Therefore, every solution of Eq. (1) is oscillatory.

We can easily see that the following result is true.

Theorem 2.2. If there exists a positive, nondecreasing function $\rho(t) \in C^{1}\left(\left[t_{0}, \infty\right)\right)$, such that for any constant $M>0$,

$$
\limsup _{t \rightarrow \infty} \int_{l}^{t}\left[H(s, l) \rho(s) C_{1}(s)-\left(h_{1}(s, l)+\sqrt{H(s, l)} \frac{\rho^{\prime}(s)}{\rho(s)}\right)^{\alpha+1} \frac{C_{2}(s)}{H^{\frac{\alpha-1}{2}}(s, l)}\right] d s>0
$$

and

$$
\limsup _{t \rightarrow \infty} \int_{l}^{t}\left[H(t, s) \rho(s) C_{1}(s)-\left(h_{2}(t, s)+\sqrt{H(t, s)} \frac{\rho^{\prime}(s)}{\rho(s)}\right)^{\alpha+1} \frac{C_{2}(s)}{H^{\frac{\alpha-1}{2}}(t, s)}\right] d s>0
$$

hold, where $C_{1}(t), C_{2}(t)$ is defined as in Theorem 2.1, then every solution of Eq. (1) is oscillatory.

Proof. For any $T \geq t_{0}$, let $a_{n}=T$, in (23) we choose $l=a_{n}$, then there exist $c_{n}>a_{n}$ such that

$\int_{a_{n}}^{c_{n}}\left[H\left(s, a_{n}\right) \rho(s) C_{1}(s)-\left(h_{1}\left(s, a_{n}\right)+\sqrt{H\left(s, a_{n}\right)} \frac{\rho^{\prime}(s)}{\rho(s)}\right)^{\alpha+1} \frac{C_{2}(s)}{H^{\frac{\alpha-1}{2}}\left(s, a_{n}\right)}\right] d s>0$.

In (24) we choose $l=c_{n}$, then there exist $b_{n}>c_{n}$ such that

$$
\int_{c_{n}}^{b_{n}}\left[H\left(b_{n}, s\right) \rho(s) C_{1}(s)-\left(h_{2}\left(b_{n}, s\right)+\sqrt{H\left(b_{n}, s\right)} \frac{\rho^{\prime}(s)}{\rho(s)}\right)^{\alpha+1} \frac{C_{2}(s)}{H^{\frac{\alpha-1}{2}}\left(b_{n}, s\right)}\right] d s>0 .
$$


Combining (25) and (26) we obtain (10). The conclusion thus comes from Theorem 2.1. the proof is complete.

Remark If we take $p(t)=0, n=2, m=1, f(x)=|x|^{\alpha-1} x$, then Theorem 2.1 and Theorem 2.2 reduce to Theorem 2.1 and Theorem 2.2 of $\mathrm{Li}$ [7], respectively. If $r(t)=1, n=2, \alpha=1$, then Theorem 2.1 and Theorem 2.2 reduce to Theorem 2.1 and Theorem 2.2 of Zhuang and $\mathrm{Li}$ [19], respectively. For the case where $H:=H(t-s) \in X$, we have $h_{1}(t-s)=h_{2}(t-s)$ and denote them by $h(t-s)$. The subclass of $X$ containing such $H(t-s)$ is denoted by $X_{1}$, applying Theorem 2.1 to $X_{1}$ we obtain the following theorem.

Theorem 2.3. If for each $T \geq t_{0}$ and any constant $M>0$, there exists a positive, nondecreasing function $\rho(t) \in C^{1}\left(\left[t_{0}, \infty\right)\right), H \in X_{1}$ and $a_{n}, c_{n} \in R$ such that $T \leq$ $a_{n}<c_{n}$ and

$$
\begin{aligned}
& \int_{a_{n}}^{c_{n}} H\left(s-a_{n}\right)\left[\rho(s) C_{1}(s)+\rho\left(2 c_{n}-s\right) C_{1}\left(2 c_{n}-s\right)\right] d s \\
> & \int_{a_{n}}^{c_{n}}\left[h\left(s-a_{n}\right)+\sqrt{H\left(s-a_{n}\right)} \frac{\rho^{\prime}(s)}{\rho(s)}\right]^{\alpha+1} \frac{C_{2}(s)}{H^{\frac{\alpha-1}{2}}\left(s-a_{n}\right)} d s \\
+ & \int_{a_{n}}^{c_{n}}\left[h\left(s-a_{n}\right)+\sqrt{H\left(s-a_{n}\right)} \frac{\rho^{\prime}\left(2 c_{n}-s\right)}{\rho\left(2 c_{n}-s\right)}\right]^{\alpha+1} \frac{C_{2}\left(2 c_{n}-s\right)}{H^{\frac{\alpha-1}{2}}\left(s-a_{n}\right)} d s
\end{aligned}
$$

hold, where $C_{1}(t), C_{2}(t)$ is defined as in Theorem 2.1, then Eq. (1) is oscillatory.

Proof. Let $b_{n}=2 c_{n}-a_{n}$, then $H\left(b_{n}-c_{n}\right)=H\left(c_{n}-a_{n}\right)=H\left(\frac{b_{n}-a_{n}}{2}\right)$ and for any $g \in L\left[a_{n}, b_{n}\right]$,we have $\int_{c_{n}}^{b_{n}} g(s) d s=\int_{a_{n}}^{c_{n}} g\left(2 c_{n}-s\right) d s$, hence,

$$
\begin{gathered}
\int_{c_{n}}^{b_{n}} H\left(b_{n}-s\right) \rho(s) C_{1}(s) d s=\int_{a_{n}}^{c_{n}} H\left(s-a_{n}\right) \rho\left(2 c_{n}-s\right) C_{1}\left(2 c_{n}-s\right) d s, \\
\quad \int_{c_{n}}^{b_{n}}\left(h_{2}\left(b_{n}-s\right)+\sqrt{H\left(b_{n}-s\right)} \frac{\rho^{\prime}(s)}{\rho(s)}\right)^{\alpha+1} \frac{C_{2}(s)}{H^{\frac{\alpha-1}{2}}\left(b_{n}-s\right)} d s \\
=\int_{a_{n}}^{c_{n}}\left(h\left(s-a_{n}\right)+\sqrt{H\left(s-a_{n}\right)} \frac{\rho^{\prime}\left(2 c_{n}-s\right)}{\rho\left(2 c_{n}-s\right)}\right)^{\alpha+1} \frac{C_{2}\left(2 c_{n}-s\right)}{H^{\frac{\alpha-1}{2}}\left(s-a_{n}\right)} d s .
\end{gathered}
$$

So that (27) holds implies that (10) holds for $H \in X_{1}$, and therefore, Eq. (1) is oscillatory by Theorem 2.1 .

From above oscillation criteria, we can obtain different sufficient conditions for oscillation of all solutions of Eq. (1) by different choices of $H(t, s)$. Now we 
choose $H(t, s)=(t-s)^{\lambda}, t \geq s \geq t_{0}$, where $\lambda>\alpha$ is a constant. Then $H \in X_{1}$ and $h(t-s)=\lambda(t-s)^{\frac{\lambda}{2}-1}$, based on the above results we obtain the following corollary.

Corollary 2.1. Every solution of Eq. (1) is oscillatory provided that for any constant $M>0$, there exist a positive, nondecreasing function $\rho(t) \in C^{1}\left(\left[t_{0}, \infty\right)\right)$ such that for each $l \geq t_{0}$ and for some $\lambda>\alpha$, the following two inequalities hold:

$$
\begin{aligned}
& \limsup _{t \rightarrow \infty} \frac{1}{t^{\lambda-\alpha}} \int_{l}^{t}\left[(s-l)^{\lambda} \rho(s) C_{1}(s)-C_{2}(s)(s-l)^{\lambda-\alpha-1}\left(\lambda+\frac{\rho^{\prime}(s)}{\rho(s)}(s-l)\right)^{\alpha+1}\right] d s>0, \\
& \limsup _{t \rightarrow \infty} \frac{1}{t^{\lambda-\alpha}} \int_{l}^{t}\left[(t-s)^{\lambda} \rho(s) C_{1}(s)-C_{2}(s)(t-s)^{\lambda-\alpha-1}\left(\lambda+\frac{\rho^{\prime}(s)}{\rho(s)}(t-s)\right)^{\alpha+1}\right] d s>0 .
\end{aligned}
$$

where $C_{1}(t), C_{2}(t)$ is defined as in Theorem 2.1.

Define

$$
R(t)=\int_{l}^{t} \frac{d s}{r^{\frac{1}{\alpha}}(s)}, t \geq l \geq t_{0}
$$

and let

$$
H(t, s)=[R(t)-R(s)]^{\lambda}, t \geq t_{0},
$$

where $\lambda>\alpha$ is constant.

If we take $\rho(t)=1$, then by Theorem 2.2 we have the following important oscillation criterion.

Theorem 2.1. Assume that $\lim _{t \rightarrow \infty} R(t)=\infty$, then every solution of Eq.(1.1) is oscillatory provided that for any constant $M>0$, there exist a positive, nondecreasing function $\rho(t) \in C^{1}\left(\left[t_{0}, \infty\right)\right)$ such that for each $l \geq t_{0}$ and for some $\lambda>\alpha$, the following two inequalities hold:

$\limsup _{t \rightarrow \infty} \frac{1}{R^{\lambda-\alpha}(t)} \int_{l}^{t}\left[(R(s)-R(l)]^{\lambda} C_{1}(s)\left(\sigma^{\prime}(s) \sigma^{n-2}(s)\right)^{\alpha} d s>\frac{\lambda^{\alpha+1}}{M^{\alpha}(\alpha+1)^{\alpha+1}(\lambda-\alpha)}\right.$,

and

$\limsup _{t \rightarrow \infty} \frac{1}{R^{\lambda-\alpha}(t)} \int_{l}^{t}\left[(R(t)-R(s)]^{\lambda} C_{1}(s)\left(\sigma^{\prime}(s) \sigma^{n-2}(s)\right)^{\alpha} d s>\frac{\lambda^{\alpha+1}}{M^{\alpha}(\alpha+1)^{\alpha+1}(\lambda-\alpha)}\right.$,

where $C_{1}(t), C_{2}(t)$ is defined as in Theorem 2.1.

Proof. By assumption, we have

$$
h_{1}(t, s)=h_{2}(t, s)=\lambda\left[(R(t)-R(s)]^{\frac{\lambda-2}{2}} \frac{1}{r^{\frac{1}{\alpha}}(t)},\right.
$$


noting that

$$
\begin{aligned}
& \int_{l}^{t} \frac{h_{1}(s, l)^{\alpha+1} C_{2}(s)}{H^{\frac{\alpha-1}{2}}(s, l)}\left(\sigma^{\prime}(s) \sigma^{n-2}(s)\right)^{\alpha} d s=\int_{l}^{t} \frac{\lambda^{\alpha+1}\left[(R(s)-R(l)]^{\lambda-\alpha-1} r(s)\right.}{r^{\frac{\alpha+1}{\alpha}}(s) M^{\alpha}(\alpha+1)^{\alpha+1}} \\
= & \frac{\lambda^{\alpha+1}\left[(R(t)-R(l)]^{\lambda-\alpha}\right.}{(\lambda-\alpha) M^{\alpha}(\alpha+1)^{\alpha+1}},
\end{aligned}
$$

and

$$
\begin{aligned}
& \int_{l}^{t} \frac{h_{2}(t, s)^{\alpha+1} C_{2}(s)}{H^{\frac{\alpha-1}{2}}(t, s)}\left(\sigma^{\prime}(s) \sigma^{n-2}(s)\right)^{\alpha} d s=\int_{l}^{t} \frac{\lambda^{\alpha+1}\left[(R(t)-R(s)]^{\lambda-\alpha-1} r(s)\right.}{r^{\frac{\alpha+1}{\alpha}}(s) M^{\alpha}(\alpha+1)^{\alpha+1}} \\
= & \frac{\lambda^{\alpha+1}\left[(R(t)-R(l)]^{\lambda-\alpha}\right.}{(\lambda-\alpha) M^{\alpha}(\alpha+1)^{\alpha+1}},
\end{aligned}
$$

in view of $\lim _{t \rightarrow \infty} R(t)=\infty$, we have

$\lim _{t \rightarrow \infty} \frac{1}{R^{\lambda-\alpha}(t)} \int_{l}^{t} \frac{h_{1}(s, l)^{\alpha+1} C_{2}(s)}{H^{\frac{\alpha-1}{2}}(s, l)}\left(\sigma^{\prime}(s) \sigma^{n-2}(s)\right)^{\alpha} d s=\frac{\lambda^{\alpha+1}}{M^{\alpha}(\alpha+1)^{\alpha+1}(\lambda-\alpha)}$,

and

$\lim _{t \rightarrow \infty} \frac{1}{R^{\lambda-\alpha}(t)} \int_{l}^{t} \frac{h_{2}(t, s)^{\alpha+1} C_{2}(s)}{H^{\frac{\alpha-1}{2}}(t, s)}\left(\sigma^{\prime}(s) \sigma^{n-2}(s)\right)^{\alpha} d s=\frac{\lambda^{\alpha+1}}{M^{\alpha}(\alpha+1)^{\alpha+1}(\lambda-\alpha)}$.

From (28) and (30), we have that

$$
\begin{gathered}
\limsup _{t \rightarrow \infty} \frac{1}{R^{\lambda-\alpha}(t)} \int_{l}^{t}\left[(R(s)-R(l))^{\lambda} C_{1}(s)-\frac{h_{1}(s, l)^{\alpha+1} C_{2}(s)}{H^{\frac{\alpha-1}{2}}(s, l)}\right]\left(\sigma^{\prime}(s) \sigma^{n-2}(s)\right)^{\alpha} d s= \\
\limsup _{t \rightarrow \infty} \frac{1}{R^{\lambda-\alpha}(t)} \int_{l}^{t}(R(s)-R(l))^{\lambda} C_{1}(s)\left(\sigma^{\prime}(s) \sigma^{n-2}(s)\right)^{\alpha} d s-\frac{\lambda^{\alpha+1}}{M^{\alpha}(\alpha+1)^{\alpha+1}(\lambda-\alpha)}>0,
\end{gathered}
$$

i.e., (23) holds. Similarly, (29) and (31) imply that (24) holds. By Theorem 2.2, every solution of Eq. (1) is oscillatory.

This complete the proof.

Example Consider the following equation :

$$
\begin{aligned}
& {\left[\left|\left(x(t)+\left(1-e^{-\mu t}\right) x(t-\pi)\right)^{(n-1)}\right|^{\alpha-1}\left(x(t)+\left(1-e^{-\mu t}\right) x(t-\pi)\right)^{(n-1)}\right]^{\prime} } \\
+ & \frac{\beta}{t^{\alpha(n-1)+1}} e^{\gamma \alpha \mu t}|x(\gamma t)|^{\alpha-1} x(\gamma t)=0, t \geq 1,
\end{aligned}
$$


where $n \geq 2$ is even and $\alpha>0, \beta>0, \mu \geq 0,0<\gamma \leq 1$.

Here $p(t)=1-e^{-\mu t}, q(t)=\frac{\beta e^{\gamma \alpha \mu t}}{t^{\alpha(n-1)+1}}, m=1, \sigma_{1}(t)=\gamma t$.

Then $R(t)=\int_{1}^{t} d t=t-1, R^{\prime}(t)=1, \lim _{t \rightarrow \infty} R(t)=\infty, \sigma_{1}^{\prime}(t)=\gamma$, if $0<\gamma \leq \frac{1}{2}$, then $\sigma(t)=\gamma t$. if $\frac{1}{2}<\gamma \leq 1$, then $\sigma(t)=\frac{t}{2}$.

For $\rho(t) \equiv 1, \lambda>\alpha$. If $0<\gamma \leq \frac{1}{2}$, then $\sigma(t)=\sigma_{1}(t)=\gamma t$,

$$
\begin{aligned}
& \lim _{t \rightarrow \infty} \frac{1}{R^{\lambda-\alpha}(t)} \int_{l}^{t}\left[(R(s)-R(l)]^{\lambda} C_{1}(s)\left(\sigma^{\prime}(s) \sigma^{n-2}(s)\right)^{\alpha} d s\right. \\
= & \lim _{t \rightarrow \infty} \frac{1}{(t-1)^{\lambda-\alpha}} \int_{l}^{t}(s-l)^{\lambda} \frac{\beta e^{\lambda \alpha \mu s}}{s^{\alpha(n-1)+1}}(1-p(\gamma s))^{\alpha}\left(\gamma(\gamma s)^{n-2}\right)^{\alpha} d s \\
= & \lim _{t \rightarrow \infty} \frac{1}{(t-1)^{\lambda-\alpha}} \int_{l}^{t}(s-l)^{\lambda} \frac{\beta \gamma^{\alpha(n-1)}}{s^{\alpha+1}} d s \\
= & \lim _{t \rightarrow \infty} \frac{(t-l)^{\lambda}}{(\lambda-\alpha)(t-1)^{\lambda-\alpha-1}} \frac{1}{t^{\alpha+1}} \beta \gamma^{\alpha(n-1)}=\frac{\beta \gamma^{\alpha(n-1)}}{\lambda-\alpha} .
\end{aligned}
$$

Next, we will prove that

$$
\begin{aligned}
& \int_{l}^{t}\left[(R(t)-R(s)]^{\lambda} C_{1}(s)\left(\sigma^{\prime}(s) \sigma^{n-2}(s)\right)^{\alpha} d s\right. \\
\geq & \int_{l}^{t}\left[(R(s)-R(l)]^{\lambda} C_{1}(s)\left(\sigma^{\prime}(s) \sigma^{n-2}(s)\right)^{\alpha} d s .\right.
\end{aligned}
$$

Let

$$
\begin{aligned}
& G(t)=\int_{l}^{t}\left\{\left[(R(t)-R(s)]^{\lambda}-\left[(R(s)-R(l)]^{\lambda}\right\} C_{1}(s)\left(\sigma^{\prime}(s) \sigma^{n-2}(s)\right)^{\alpha} d s\right.\right. \\
= & \int_{l}^{t}\left\{(t-s)^{\lambda}-(s-l)^{\lambda}\right\} \frac{\beta}{s^{\alpha(n-1)+1} e^{\mu \alpha \gamma s}} \gamma^{\alpha(n-1)} s^{\alpha(n-2)} d s \\
= & \beta \gamma^{\alpha(n-1)} \int_{l}^{t}\left\{(t-s)^{\lambda}-(s-l)^{\lambda}\right\} \frac{1}{s^{\alpha+1} e^{\mu \alpha \gamma s}} d s
\end{aligned}
$$

then $G(l)=0$, and for $t \geq l$,

$$
\begin{aligned}
& G^{\prime}(t)=\beta \gamma^{\alpha(n-1)} \int_{l}^{t} \lambda(t-s)^{\lambda-1} \frac{1}{s^{\alpha+1} e^{\mu \alpha \gamma s}} d s-(t-l)^{\lambda} \frac{1}{t^{\alpha+1} e^{\mu \alpha \gamma t}} \\
\geq & \beta \gamma^{\alpha(n-1)} \frac{1}{t^{\alpha+1} e^{\mu \alpha \gamma t}}\left[\int_{l}^{t} \lambda(t-s)^{\lambda-1} d s-(t-l)^{\lambda}\right] \\
= & \frac{\beta \gamma^{\alpha(n-1)}}{t^{\alpha+1} e^{\mu \alpha \gamma t}}\left[-\left.(t-s)^{\lambda}\right|_{l} ^{t}-(t-l)^{\lambda}\right]=0 .
\end{aligned}
$$


Hence $G(t) \geq G(l)=0$ for $t \geq l$, i.e., (2.31) holds. By (34) and (35), we have

$$
\lim _{t \rightarrow \infty} \frac{1}{(t-1)^{\lambda-\alpha}} \int_{l}^{t}\left[(R(t)-R(s)]^{\lambda} C_{1}(s)\left(\sigma^{\prime}(s) \sigma^{n-2}(s)\right)^{\alpha} d s>\frac{\beta \gamma^{\alpha(n-1)}}{\lambda-\alpha} .\right.
$$

Then for $\beta>\frac{\left(\frac{\alpha}{\alpha+1}\right)^{\alpha+1}}{M^{\alpha} \gamma^{\alpha(n-1)}}$, there exists $\lambda>\alpha$ such that

$$
\frac{M^{\alpha} \gamma^{\alpha(n-1)} \beta}{\lambda-\alpha}>\frac{\lambda^{\alpha+1}}{(\lambda-\alpha)(\alpha+1)^{\alpha+1}}>\frac{\alpha^{\alpha+1}}{(\lambda-\alpha)(\alpha+1)^{\alpha+1}},
$$

this means that

$$
\frac{\gamma^{\alpha(n-1)} \beta}{\lambda-\alpha}>\frac{\lambda^{\alpha+1}}{M^{\alpha}(\lambda-\alpha)(\alpha+1)^{\alpha+1}},
$$

so that (28) and (29) hold for the same $\lambda$. Applying Theorem 2.4, we fined (33) is oscillatory for $\beta>\frac{\left(\frac{\alpha}{\alpha+1}\right)^{\alpha+1}}{M^{\alpha} \gamma^{\alpha(n-1)}}$. If $\frac{1}{2}<\gamma \leq 1$, then $\sigma(t)=\frac{t}{2}$, use the same method above, we can get (33) is oscillatory for $\beta>\frac{\left(\frac{\alpha}{\alpha+1}\right)^{\alpha+1}}{M^{\alpha}\left(\frac{1}{2}\right)^{\alpha(n-1)}}$. However, the main results of $[2,15]$ fail to apply to $(33)$, since $\mu \neq 0$.

\section{REFERENCES}

1. R.P. Agarwal, S.H. Shieh, C.C. Yeh, "Oscillation criteria for second Order retarded differential equations", Math.Comput. Modelling 26 (1997), 1-11.

2. R.P. Agarwal, S.R. Grace and D. O'Regan, "Oscillation criteria for certain $n$-th order differential equations with deviating arguments", J. Math. Anal. Appl. 262 (2001), 601-622.

3. J.L. Chern, W.Ch. LiAn, C.C. YeH, "Oscillation criteria for second order half-linear differential equations with functional arguments", Publ.Math.Ddbrecen 48 (1996), 209216.

4. J. Dzurina And I.P. Stavroulakis, "Oscillation criteria for second-order delay differential equations", Applied Mathematics and Computation 140 (2003), 445-453.

5. A. Elbert, "A half-linear second order differential equation", Colloquia Math. Soc. Janos Bolyai, Qualitative Theory of differential equations 30 (1979), 153-180.

6. A. Elbert, "Oscillation and nonoscillation theorems for some nonlinear differential equations, in: Ordinary and Partial Differential Equations", Lecture Notes in Mathematics 964 (1982), 187-212.

7. W.T. LI, "Interval oscillation of second order half-linear functional differential equations", Appl. Math. Comput. 155 (2004), 451-468. 
8. F. Meng And R. Xu, "Oscillation Criteria for Certain Even Order Quasi-Linear Neutral Differential Equations with Deviating Arguments", Appl. Math. Comput. 190 (2007), 458-464.

9. F. Meng And R. Xu, "Kamenev-type Oscillation Criteria for Even Order Neutral Differential Equations with Deviating Arguments", Appl. Math. Comput. 190 (2007), $1402-1408$.

10. D.D. Mirzov, "On the oscillation of system of nonlinear differential equations", Diferencianye Uravnenija 9 (1973), 581-583.

11. D.D. Mirzov, "On some analogs of Sturm's and Kneser's theorems for nonlinear systems", J. Math. Anal. Appl. 53 (1976), 418-425.

12. D.D. Mirzov, "On the oscillation of solutions of a system of differential equations", Mat. Zametki 78 (1978), 401-404.

13. CH.G. Philos, "A new criteria for the oscillatory and asymptotic behavior of delay differential equations", Bull. Acad. Pol. Sci. Ser. Mat. 39 (1981), 61-64.

14. Y.G. Sun And F.W. Meng, "Note on the paper of Dzurina and Stavroulakis", Appl. Math. Comput. 174 (2006), 1634-1641.

15. Z. XU, Y. XIA, "Integral averaging technique and oscillation of certin even order delay differential equations", J. Math. Anal. Appl. 292 (2004), 238-246.

16. R. Xu, F. Meng, "Some New Oscillation Criteria For Second Order Quasi-Linear Neutral Delay Differential Equations", Appl. Math. Comput. 182 (2006), 797-803.

17. R. Xu, F. Meng, "New Kamenev-type oscillation criteria for second order neutral nonlinear differential equations", Appl. Math. Comput. 188 (2007), 1364-1370.

18. R. Xu, F. Meng, "Oscillation Criteria for Second Order Quasi-Linear Neutral Delay Differential Equations", Appl. Math. Comput. 192 (2007), 216-222.

19. R.K. ZhANG, W.T. LI, "Interval oscillation criteria for second order quasilinear perturbed differential equations", Int.J.Differential Equations Appl. 7:2 (2003), 165-179.

Run Xu: Department of Mathematics, Qufu Normal University, Qufu, 273165, Shangdong, China.

FAnwei Meng: Department of Mathematics, Qufu Normal University, Qufu, 273165, Shangdong, China. 